Relations industrielles

Industrial Relations

\title{
La dispersion au travail, Par Caroline Datchary, Toulouse : Octarès éditions, 2011, 192 p., ISBN : 978-2-915346-88-6.
}

\section{Sébastien Bruère}

Volume 67, numéro 4, automne 2012

URI : https://id.erudit.org/iderudit/1013204ar

DOI : https://doi.org/10.7202/1013204ar

Aller au sommaire du numéro

Éditeur(s)

Département des relations industrielles de l’Université Laval

ISSN

0034-379X (imprimé)

1703-8138 (numérique)

Découvrir la revue

Citer ce compte rendu

Bruère, S. (2012). Compte rendu de [La dispersion au travail, Par Caroline Datchary, Toulouse : Octarès éditions, 2011, 192 p., ISBN : 978-2-915346-88-6.]

Relations industrielles / Industrial Relations, 67(4), 729-731.

https://doi.org/10.7202/1013204ar

Tous droits réservés @ C Département des relations industrielles de l’Université Laval, 2012
Ce document est protégé par la loi sur le droit d'auteur. L'utilisation des services d'Érudit (y compris la reproduction) est assujettie à sa politique d'utilisation que vous pouvez consulter en ligne.

https://apropos.erudit.org/fr/usagers/politique-dutilisation/ 
tions circumstances and current challenges; and, at the end of each chapter, a tabular chronology of employment-relations milestones. Being the 5 th book in a series, the editors clearly intended to bring the work up to date. They fully accomplished this goal. Hence, at least when I read the book, key issues were topical and many were still being dealt with in the press and other less serious media. Indeed, as I read the book I was again reminded that, at least insofar as employment relations is concerned, serious inquirers need to eschew popular print and electronic media if they want to understand the underlying and/or more conceptual element of a phenomena.

Aside from the book's consistent structure, it is uncanny that, despite its multiple contributors, stylistic aspects of its written expression are very similar. This perhaps reflects the editors' substantial involvement in redrafting. Whatever the case, the work is clear and straightforward throughout. For example, sentences are mostly short and concise. Despite the fact that material being dealt with is often complex, the authors take care to be concise and differentiate between salient and less relevant material. Without exception, it is possible to effortlessly follow the narrative of each chapter. Along the way, key employment relations themes and challenges are typically well differentiated from mere description. Such a style creates a sense of methodological discipline and has a great deal of instructional value (those of us fixated on methods mostly make a big deal about the observation/inference distinction but often fall short with our examples).

My criticisms of the book are very minor and mostly concerned with quality control. For example, the work contains a few typos and - sometimes $-I$ think that conclusions do not follow easily from the data upon which they are based. An example of this latter problem is on pages 92-93 dealing with (alleged) declining unemployment in Canada following the country's 2000 natu- ral resources boom. Table 4.1's data (p. 93) do not show any particular trend towards declining unemployment in the post-2000 period. Furthermore, it is noted at the end of page 91 that "Canada achieved a 32 year low in unemployment in 2007 with a rate of 5.8 percent." In fact Table 4.1 indicates that the rate of unemployment in that year is 6.0 percent. There are a couple of similar problems in other sections of the work.

In their latest work, Bamber, Lansbury and Wailes have made a serious attempt to achieve two seemingly incompatible or at least mismatched - goals. First, they have managed to give a detailed (and strategic) overview of modern comparative employment relations. Second, and perhaps more importantly, they have mostly made the narrative interesting and accessible. I reiterate, doing these things simultaneously is perhaps one of the toughest challenges in our field. For this reason alone, I conclude that this work is an important milestone. It should be on the shelf (or downloaded to the e-reader) of all serious $21^{\text {st }}$ century employment relations scholars.

\section{Anthony Gould}

Université Laval

\section{La dispersion au travail}

Par Caroline Datchary, Toulouse : Octarès éditions, 2011, 192 p., ISBN : 978-2-91534688-6.

Bien qu'il s'agisse d'un ouvrage ayant, avant tout, comme objet la description sociologique d'un certain type de situations de travail, il porte un regard nouveau sur les transformations du travail et sur les questions que soulèvent le stress et la pression des postes impliquant des responsabilités. Élargissant son approche, issue de la théorie des conventions et plus particulièrement de la sociologie des engagements, par celle de l'activité de travail qui est historiquement présente dans la sociologie du travail française, Caroline Datchary nous emmène à la découverte des situations de travail dispersives. II s'agit de ces situations où la 
personne est fréquemment confrontée à des engagements multiples dans un espace temporel restreint.

Dans un premier chapitre, l'auteure constate l'absence de visibilité de ces situations de travail et cherche, par conséquent, à mieux les définir. Cette invisibilité serait le fruit, d'une part, d'une norme " attendue » de réactivité de la part des salariés au sein de l'organisation, mais aussi du fait que la dispersion est souvent associée aux troubles de l'attention ou à l'hyperactivité, ce qui ne correspond pas à l'objet que souhaite traiter l'auteure. Cela l'oblige à définir une nouvelle catégorie, à savoir ces fameuses situations de dispersion ou situations de travail dispersives. Pour évaluer le sens de cette catégorie descriptive, I'auteure a fait le choix d'élargir son approche et de construire une démarche ethnographique comparative, centrée sur l'activité, dont quatre cas sont plus particulièrement exposés dans l'ouvrage. Chacun des cas peut permettre au lecteur de le rapprocher d'un type d'emploi particulier et d'en tirer pour lui-même les conséquences, en termes de conception ou d'organisation du travail, que ces activités professionnelles peuvent nécessiter.

Le premier cas est celui du conducteur de travaux. De nombreux textes traitent de la spécificité des chantiers de construction et du fait que souvent l'organisation du travail se dessine davantage dans l'improvisation que dans la planification. L'auteure relève ainsi plusieurs caractéristiques de la dispersion de cette fonction parmi lesquelles : la polyvalence, I'aspect distribué de I'activité entre plusieurs chantiers et enfin le rythme de travail saccadé. Cependant, ce qui retient surtout l'attention, ce sont les stratégies que développent les acteurs pour prévenir les situations de dispersion. Le conducteur de travaux privilégie les échanges en face-à-face avec les ouvriers directement sur le chantier et délaisse les outils informatiques pour les échanges. Ce faisant, il réalise un important travail d'organisation, en utilisant des stratégies lui permettant de réduire la variabilité qu'il pourrait rencontrer.

Le second cas concerne le trader, ce métier d'opérateur financier qui doit gérer un nombre important d'informations à chaque instant. Le premier constat de l'auteure, par rapport à cette profession, est l'importance des moyens techniques mis à la disposition de l'opérateur pour gérer les situations de dispersion. Au-delà de ces éléments, I'expérience et le tour de main des acteurs jouent également un rôle dans la gestion de la dispersion en leur permettant de se construire plus facilement, à chaque instant, des schémas d'interprétation de l'information pour agir de manière efficiente.

Le troisième cas aborde, quant à lui, la fonction de manager. À son sujet, l'auteure constate d'abord qu'il faut dépasser I'analyse classique de la littérature qui ne concerne, en grande partie, que les situations d'interruption. Si elle constate le fait que les activités s'enchevêtrent, elle observe aussi que ces interruptions peuvent être associées tant à un coût qu'à un effet bénéfique. Elle remarque également deux éléments particuliers créateurs de situations dispersives: la présence des dispositifs de messagerie et la sollicitation continuelle que la présence des messages engendre. Là aussi, I'auteure observe tout un travail qui permet au manager de gérer les sollicitations tout en préservant son activité, qui passe par l'établissement de conventions, le paramétrage des outils ou encore par l'usage de support papier. Dans le même temps, I'auteure constate un travail d'adaptation face aux sollicitations. Ce dernier se manifeste autant par des ajustements de la posture du corps, que par une gestion des priorités et du temps. Cet effort d'accommodation est d'autant plus visible lorsqu'il ne fonctionne plus, c'est-à-dire lorsque les situations dispersives engendrent des situations non maîtrisées ou en surcharge.

Enfin, le dernier cas est celui d'une agence gérant l'organisation de spectacles et d'évènements. Ce cas particulier permet 
de mettre l'accent sur les situations qui ont des limites de temps parfois très courtes et qui sont gérées de manière collective. La question du temps, dans les agences évènementielles, semble avoir des effets à la fois sur l'organisation des activités et sur les émotions. De plus, la dimension collective joue à plusieurs niveaux. Tout d'abord, il y a une dimension de jeu de pouvoir lors de la constitution du collectif, qui doit permettre la gestion collective de la dispersion et de ses effets. Toutefois, cela pose un enjeu particulier à l'insertion dans le collectif de travail et donne une plus grande importance au travail communicationnel. Ce travail, qui va soit faciliter la gestion des situations dispersives ou, au contraire, la rendre plus difficile, se déploie à deux niveaux. Premièrement, il est dirigé vers les collègues et nécessite beaucoup d'énergie du fait de la situation de mobilité dans laquelle chacun des acteurs se trouve. Deuxièmement, il est axé vers la relation client. Or le type de client et la forme que cette relation prend, en termes d'engagement, ont un effet important sur la fréquence d'apparition de situations dispersives.

Bien que l'auteure ait observé une diversité de situations de travail dispersives, elle constate des activités similaires dans chacun des cas, ce qui lui permet de formuler plusieurs apports sociologiques. Tout d'abord, il y a le constat de l'ambivalence des outils informatiques, qui sont à la fois aide à la gestion des situations dispersives et source de dispersion. II y a aussi les questions de coopération, qui s'avèrent à la fois complexes et nécessaires pour réaliser cette gestion. Enfin, son étude fait ressortir deux dimensions davantage individuelles. D'une part, l'importance de l'adéquation entre les compétences et les charges amène des réflexions, tant sur la formation que sur l'environnement de travail. D'autre part, du fait que la gestion de la dispersion nécessite un travail d'intégration de plusieurs engagements hétérogènes, la question de la consistance de la personne prend une importance particulière, c'est- à-dire que le travailleur, aux prises avec les situations de dispersion, doit faire un effort particulier pour articuler l'ensemble de ses actions. Il doit se coordonner lui-même, sous peine de paraître comme changeant d'idée régulièrement et non sincère vis-à-vis de ses interlocuteurs.

En terminant, cet ouvrage aborde la question de la dispersion au travail sous I'angle de l'activité. En choisissant cette approche, Caroline Datchary ouvre une nouvelle avenue qui lui permet de poser un regard neuf sur un ensemble de métiers et de situations de travail susceptibles d'engendrer de la dispersion. Bien que ne traitant pas explicitement des questions plus globales de santé au travail que ses travaux soulèvent, elle pose un premier jalon, précis et utile, à la compréhension de l'activité nécessaire face à certaines situations de travail. Pour l'instant, charge est laissée au lecteur d'en tirer les conclusions pour l'agir organisationnel ou les innovations sociales que cette activité nécessiterait de mettre en place ou de concevoir dans les entreprises.

\section{Sébastien Bruère}

Université Laval

\section{Canadian Labour in Crisis: Reinventing the Workers' Movement}

By David Camfield, Halifax, N.S.: Fernwood Publishing, 2011, 168 pp., ISBN: 978-1-55266416-2.

David Camfield's Canadian Labour in Crisis: Reinventing the Workers' Movement falls within the larger literature documenting the current state of labour movements in affluent democracies. Going beyond numerical analyses of union membership and density rates, Camfield's text adds to the qualitative discussion by examining the internal and external power transformations of Canadian unions. Despite Canadian union density incurring a slow decline from its peak of 41.8 percent in 1984 to 29.5 percent in 2009, Camfield suggests that density does not translate into power (p. 14). 